\title{
Etude Comparative de l'adsorption de l'acide 5-sulfosalicylique et des ions orthophosphates en solution aqueuse par des charbons actifs
}

\author{
Joseph Ketcha Mbadcam*, Benoît Loura Benguellah**, Harouna Massai* et Patrick Hervé Tchoua Ngamou* \\ * Laboratoire de chimie physique et théorique, Faculté des sciences de l'Université de Yaoundé I, BP 8I 2, Yaoundé, Cameroun \\ Email :jketcha@yahoo.com / Jketcha@uycdc.uninet.cm \\ *** Département de chimie, Faculté des sciences, Université de Ngaoundéré, Cameroun
}

\begin{abstract}
L'adsorption de l'acide 5-sulfosalicylique et des ions orthophosphates en solution aqueuse par deux charbons actifs commerciaux a été effectuée. Les isothermes d'adsorption de Langmuir et de Freundlich ont été obtenues à $298^{\circ} \mathrm{K}$ et le calcul des capacités d'adsorption montre que les ions orthophosphates sont mieux adsorbés que l'acide 5-sulfosalicylique et que les deux charbons ont des propriétés d'adsorption liées au nombre de sites actifs accessibles, c'est-à-dire présents à la surface des grains.
\end{abstract}

Mots-clefs : Adsorption, ions orthophosphates, acide 5-sulfosalicylique, charbon actif, sites actifs, pourcentage de dispersion.

A study was made of the adsorption of 5-sulfosalicylic acid and orthophosphate ions from aqueous solutions by two commercially activated carbons. Adsorption isotherms obtenained at $298^{\circ} \mathrm{K}$ were both of Langmuir and Freundlich types, and from them adsorption capacities were calculated. From these results, the activated carbon with a $\mathrm{pH}=8$ adsorbs both adsorbates better than the other one $(\mathrm{pH}=7)$ and the orthophosphate ions are better adsorbed than the 5 -sulfosalicylic acid. These results are comfirmed from theoretical calculations of accessible number of active sites obtained for both the activated carbons. It has also been shown that the percentage dispersion of these active sites is high at the surface.Those results correspond with the number of active sites accessible at the surface.

Keywords: Adsorption, orthophosphate ions, 5-sulfosalicylic acid, activated carbon, active site, percentage dispersion.

\section{INTRODUCTION}

L'adsorption des molécules organiques et inorganiques par des charbons actifs constitue un processus de surface. L'adsorption implique l'accumulation ou la concentration de la substance adsorbée à la surface de l'adsorbant. Ce phénomène peut aussi avoir lieu à l'interface des deux phases, par exemple liquide-liquide, gaz-liquide, gaz-solide ou liquide-solide [I, 2, 3, 4]. L'usage de pesticides est très répandu dans le monde. Si cet usage permet des gains de production agricole, les effets négatifs sont connus sur la santé humaine [5] et sur l'écosystème. En effet, les pesticides en excès peuvent être accumulés biologiquement dans la chaîne alimentaire $[6,7]$. De plus, les effets à long terme des pesticides de faibles niveaux sur l'homme sont cause de sérieux dégâts [8]. Virtuellement, chaque usage du pesticide dans l'air, le sol ou l'eau, produit des déchets solides qui contiennent des ingrédients biodégradables et/ou des sousproduits indésirables. Les phosphates, les sulfates ainsi que leur dérivés sont parfois dans l'eau comme des produits finis de l'hydrolyse des différents pesticides organophosphoreux et organosulfureux [9]. De plus, ces phosphates et sulfates sont utilisés dans les détergents et constituent des polluants majeurs. Le charbon actif est très souvent utilisé comme adsorbant lors du processus de purification des eaux $[1-4,10,11,12]$. Les recherches antérieures ont montré que les charbons actifs d'origine agricole peuvent être utilisés pour éliminer les polluants des eaux [13-|5].

Ici, les charbons actifs commerciaux ont été utilisés parce qu'ils sont de type-H, c'est-à-dire présentent des surfaces basiques. Dans ce sens, le but de cet article est d'étudier le comportement de deux charbons actifs d'origine végétale lors du traitement des eaux usées et polluées par l'acide 5-sulfosalicylique et par les ions orthophosphates sous conditions statiques. Le nombre de sites actifs accessibles à la surface des charbons actifs a été calculé et leur pourcentage de dispersion étudié.

\section{ETUDE EXPERIMENTALE}

Dans le présent travail, deux échantillons de charbons actifs commerciaux d'origine végétale en poudre, indexés COMI et COM2 et provenant respectivement de la Société Lyonnaise d'Herboristerie (Laboratoire VITAFLOR) et de la Société PROLABO, en France, ont été utilisés.

L'adsorption de l'acide 5-sulfosalicylique et de $\mathrm{Na}_{2} \mathrm{HPO}_{4} \cdot 2 \mathrm{H}_{2} \mathrm{O}$ a été réalisée en régime statique. Les isothermes d'adsorptions ont été déterminées en utilisant des erlenmeyers contenant $0,05 \mathrm{~g} / 50 \mathrm{~mL}$ de solution de $\mathrm{Na}_{2} \mathrm{HPO}_{4} \cdot 2 \mathrm{H}_{2} \mathrm{O}$ pour l'adsorption des ions orthophosphates, et I $\mathrm{g} / 20 \mathrm{~mL}$ de solution pour l'adsorption de l'acide 5-sulfosalicylique. Ces erlens sont ensuite fermés avec des bouchons en caoutchouc et agités pendant 5 heures puis laissés au repos pendant 2 heures à la température ambiante de $298^{\circ} \mathrm{K}$. Après filtration, la concentration à l'équilibre des ions orthophosphates a été déterminée par utilisation d'un spectrophotomètre CORNING 259 à une longueur 
d'onde $\boldsymbol{\lambda}=720 \mathrm{~nm}$ suivant la méthode du bleu au molybdate [16]. Celle de l'acide 5-sulfosalicylique a été déterminée par méthode volumétrique en utilisant comme indicateur coloré la phénophtaléine. Les concentrations de soluté adsorbé sont déterminées par différence entre la concentration initiale et la concentration à l'équilibre de la solution. Les valeurs obtenues ont permis l'établissement des isothermes d'adsorption.

\section{RESULTATS ET DISCUSSION}

En suivant le mode opératoire ci-dessus, les résultats expérimentaux auxquels nous avons abouti nous ont permis de déterminer le type d'adsorption, les constantes de Freundlich $\left(K_{F}, n\right)$, la constante de Langmuir $\left(K_{L}\right)$, la quantité d'adsorption maximale (Qo), la fraction de surface du solide recouverte par les molécules adsorbées, le nombre de sites actifs accessibles à la surface du solide et la dispersion.

\section{Type d'adsorption}

Les courbes de la Figure I représentent les isothermes d'adsorption de Langmuir des ions orthophosphates et de l'acide 5-sulfosalicylique par les charbons actifs COMI et COM2. Nous constatons que ces isothermes sont toutes de type I de la classification de Langmuir. Elles montrent que COMI et COM2 sont des adsorbants microporeux (pores à faible diamètre $d<20 \AA$ ) et signalent la présence de faibles interactions à la surface des adsorbants car le nombre de couches adsorbées ne peut augmenter librement [14, I5]. L'adsorption est donc monomoléculaire et il y a saturation du solide lors du remplissage de la monocouche.

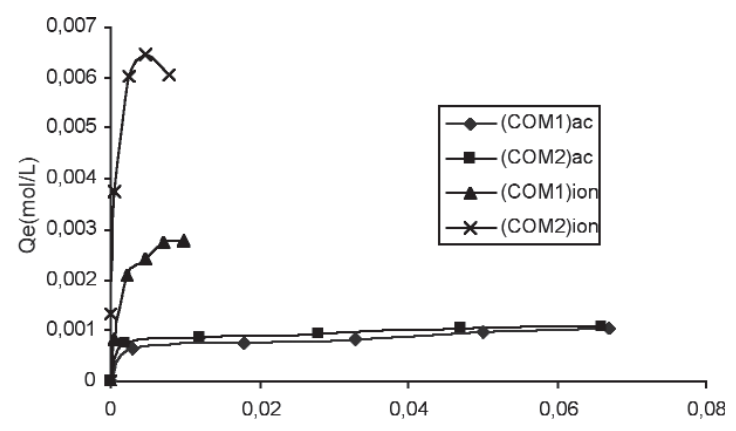

Figure 1 : Isothermes de Langmuir

où :

$\mathrm{Q}_{\mathrm{e}}=$ quantité de soluté adsorbée à l'équilibre par gramme d'adsorbant

$\mathrm{C}_{\mathrm{e}}=$ concentration de soluté à l'équilibre (COMI)

ac = charbon actif commercial pour l'acide 5-sulfosalicylique $(\mathrm{COMI})$ ion $=$ charbon actif commercial pour l'ion orthophosphate.

\section{Détermination de Q。 et KL}

Les courbes $1 / Q_{e}=f\left(I / C_{e}\right)$ de la Figure 2 étant des droites, l'adsorption obéit à la transformation expérimentale linéaire de Langmuir $\left(1 / Q_{e}=1 / Q_{0} K_{L} C_{e}+\mid / Q_{0}\right)$. En utilisant la méthode de régression linéaire, les valeurs des constantes, $Q_{0}$ et $K_{\llcorner}$ont été déduites et consignées dans le Tableau I. Nous constatons d'une part que les valeurs de la capacité maximale d'adsorption $Q_{0}$ et de KL sont plus élevées pour les ions orthophosphates que pour l'acide 5-sulfosalicylique, et d'autre part que quelle que soit la solution adsorbée, les valeurs de COM2 sont plus élevées que celles de COMI. Ceci peut être attribué au fait que la surface de COM2 est plus basique que celle de COMI. Hypothèse déduite de leurs valeurs respectives de $\mathrm{pH} 7$ et 8 , puisqu'il a été démontré que lorsque la basicité d'un charbon augmente, la capacité d'adsorption des espèces anioniques à sa surface augmente également [17].

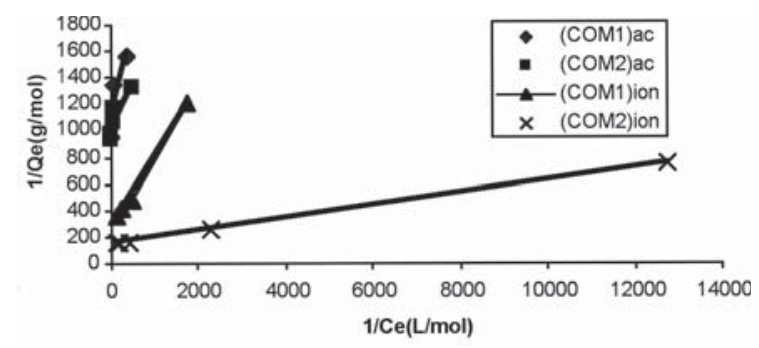

Figure 2 :Transformées linéaires de l'isotherme de Langmuir

\begin{tabular}{|c|c|c|c|c|}
\hline Charbons actifs & Solutions & $Q_{0.10^{-3}}(\mathrm{~mol} / \mathrm{g})$ & $\mathbf{K}_{\mathbf{L}}$. & $\mathbf{R}$ \\
\hline \multirow[t]{2}{*}{ COM1 } & $\Lambda$ cide 5-sulfosalicylique & 9,75 & 702 & 0,94 \\
\hline & Ions orthophosphates & 2,59 & 1015 & 0,95 \\
\hline \multirow[t]{2}{*}{$\mathrm{COM} 2$} & $\Lambda$ cide 5-sulfosalicylique & 102 & 900 & 0,96 \\
\hline & Ions orthophosphates & 4,00 & 1236 & 0,96 \\
\hline
\end{tabular}

Tableau I : Capacité d'adsorption Qo, et constante d'adsorption de Langmuir KL

On peut donc penser que COM2 présente une surface de potentiel positif par rapport à celle de COMI, et par conséquent adsorbe mieux les ions orthophosphates que COMI. Une autre hypothèse de l'adsorption facile des ions orthophosphates par rapport à l'acide 5-sulfosalicylique s'explique par la grosseur des deux molécules. L'adsorption étant monomoléculaire, seuls les micropores sont responsables. Par conséquent, les molécules des ions orthophosphates, moins volumineux, pénètrent plus facilement à l'intérieur des micropores que les molécules d'acide 5-sulfosalicylique.

Détermination des constantes d'adsorption de Freundlich $\mathrm{K}_{\mathrm{F}}$ et I/n

Les droites $\ln Q_{e}=f\left(\ln C_{e}\right)$ de la Figure 3 obtenues à partir de la transformée logarithmique de l'équation de Freundlich $\left(Q_{e}=K_{F} \cdot C_{e}^{1 / n}\right)$ ont permis de déterminer les constantes $K_{F}$ et I/n. Les valeurs obtenues sont consignées dans le Tableau 2.

\begin{tabular}{|c|c|c|c|c|}
\hline Charbons actifs & Solutions & $1 / n$ & $\mathrm{~K}_{\mathrm{r}}: 10^{-3}$ & $\mathbf{R}$ \\
\hline \multirow[t]{2}{*}{ COM1 } & $\Lambda$ cide 5-sulfosalicylique & 0,23 & 1,29 & 0,99 \\
\hline & Ions orthophosphates & 0,38 & 33,3 & 0,98 \\
\hline \multirow[t]{2}{*}{ COM2 } & $\Lambda$ cide 5-sulfosalicylique & 0,23 & 1,52 & 0,94 \\
\hline & Ions orthophosphates & 0,59 & 42,5 & 0,97 \\
\hline
\end{tabular}

Tableau 2 :Constantes d'adsorption de Freundlich $K_{F}$ et I/n

Les valeurs de $1 / n$ obtenues sont comprises entre 0,2 et 0,8 [18]. L'adsorption obéit au modèle de Freundlich et les deux charbons sont de bons adsorbants. Sachant que du point de vue de la molécule adsorbée, le charbon le plus efficace est celui 
ayant la plus grande pente (valeur de I/n la plus élevée) [1 8], nous constatons que COM2 présente des valeurs les plus élevées pour les ions orthophosphates.

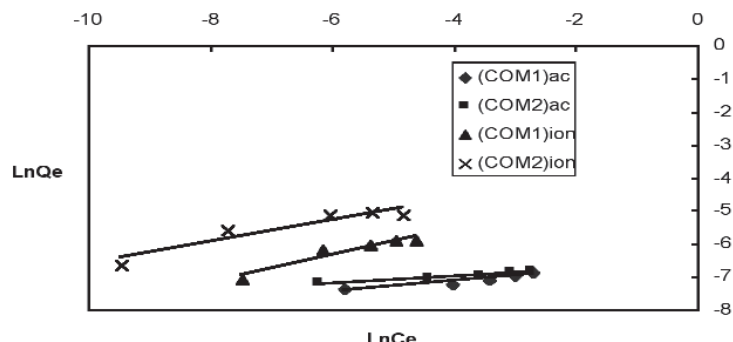

Figure 3 :Transformées linéaires de l'isotherme de Freundlich

\section{Détermination du nombre de sites actifs}

Sachant que les isothermes d'adsorption obtenues sont de type I de la classification de Langmuir, et montrant que COMI et COM2 sont des adsorbants microporeux, nous avons supposé que seuls les micropores situés à la surface de chaque charbon sont responsables de l'adsorption. Les macro et mésopores servent uniquement de voie d'accès et de communication aux micropores. II a été démontré que pour des charbons actifs microporeux, les micropores représentent environ $95 \%$ de leur surface spécifique $[19,20]$. II est donc évident que les sites actifs correspondent aux pores à la surface desquels se trouvent les molécules de l'adsorbat. Ainsi, le calcul théorique du nombre de sites actifs donne directement le nombre de sites actifs accessibles. A cet effet, nous avons supposé un modèle idéal de charbon actif où les pores sont de même type et ont la même forme (forme cylindrique), le même diamètre (diamètre moyen) et la même épaisseur (épaisseur moyenne) [20]. Sachant que le rayon des micropores varie entre 18 et $20 \AA[21]$, nous avons pris comme rayon moyen $R_{\text {moy }}=19 \AA$ et comme épaisseur moyenne $e_{\text {moy }}=1,15 \mathrm{~nm}$. Le volume moyen d'un micropore est déduit de la formule $V_{\text {moy }}=\pi \cdot R_{\text {moy }}^{2} e_{\text {moy. }}$ Connaissant la quantité d'ions orthophosphates et d'acide 5-sulfosalicylique adsorbée par gramme d'adsorbant, et en se servant de la masse volumique de $\mathrm{Na}_{2} \mathrm{HPO}_{4} \cdot 2 \mathrm{H}_{2} \mathrm{O}(\mathrm{r}=1068 \mathrm{~g} / \mathrm{L})$ et de l'acide 5-sulfosalicylique $(r=1 \mid 80 \mathrm{~g} / \mathrm{L})$, il est possible de déterminer le volume de solution adsorbée par la relation $\vee=Q_{\mathrm{e}} / \rho$. Par exemple, pour l'échantillon (COMI) ac, nous avons obtenu $Q e=10.5 .10^{-4} \mathrm{~mol} / \mathrm{g}$. En utilisant la relation précédente, nous obtenons un volume $V=8,89 \cdot 10^{-7} \mathrm{~L}$. Par conséquent, il est possible d'estimer le nombre total de sites actifs qui représente le nombre de sites actifs accessibles en utilisant la relation $\mathrm{N}=\mathrm{V} / \mathrm{V}_{\text {moy }}$. Donc $\mathrm{N}=8,89 \cdot 10^{-7} / 1,3 \cdot 10^{-26}=6,84.10^{22}$ sites $/ g$.

Ces résultats nous ont permis d'étudier la variation du nombre de sites actifs de chaque charbon en fonction de la quantité de solution adsorbée (Figure 4). Nous constatons que COM2 présente un nombre important de sites actifs par rapport à COMI, et que quel que soit le charbon utilisé, l'adsorption des ions orthophosphates présente un nombre de site moins élevé que celle de l'acide 5-sulfosalicylique. Nous pensons que plus le charbon actif est basique, plus le nombre de sites actifs accessibles est élevé et l'adsorption est forte. Car il a été démontré que lorsque la basicité d'un charbon augmente, la capacité d'adsorption des espèces anioniques à sa surface augmente également [17].

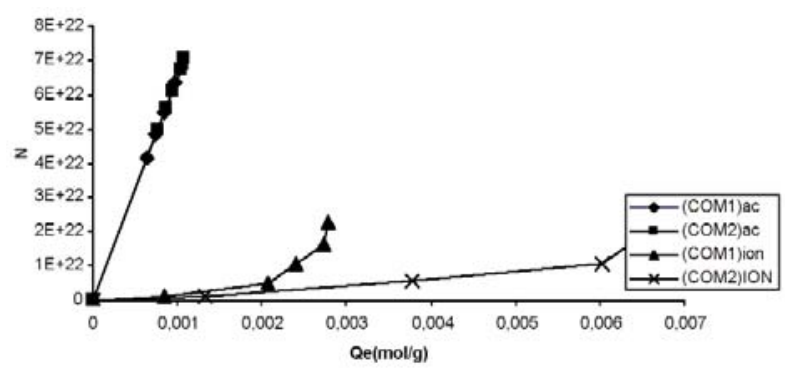

Figure 4 : Variation du nombre de sites actifs accessibles en fonction de la quantité de soluté adsorbé

\section{Dispersion des sites actifs accessibles (d\%)}

La dispersion est un paramètre important dans le processus d'adsorption. Elle nous renseigne sur la façon dont les sites actifs sont éparpillés et répartis sur la surface du charbon actif [22]. Nous pouvons donc la définir comme étant le rapport entre le nombre de sites actifs accessibles et le nombre total de sites actifs de surface, ce qui nous conduit à la relation $(I)$ :

$D=(\mathrm{N} \times / \mathrm{Nt}) 100$

où $\mathrm{Nx}=$ Nombre de sites de type $\mathrm{x}$, et $\mathrm{Nt}=$ Nombre total de sites

En appliquant la relation ci-dessus, les valeurs de dispersion obtenues ont été consignées dans le Tableau 3.

\begin{tabular}{|l|l|c|}
\hline Charbons actifs & Solutions & $\begin{array}{c}\text { Dispersion de sites actifs } \\
\text { accessibles (d\%) }\end{array}$ \\
\hline COM 1 & Acide 5-sulfosalicylique & 24 \\
\cline { 2 - 3 } & Ions orthophosphates & 51 \\
\hline \multirow{2}{*}{ COM 2 } & Acide 5-sulfosalicyliquc & 25 \\
\cline { 2 - 3 } & Ions orthophosphates & 56 \\
\hline
\end{tabular}

Tableau 3 : Dispersion des sites actifs accessibles à la surface de COMI et COM2

Nous constatons que la répartition de sites actifs accessibles augmente avec la capacité d'adsorption du charbon actif. Cette répartition augmente en fonction du nombre de sites actifs accessibles. Ainsi, plus le matériau ou les particules sont petits, plus les sites sont dispersés et plus le charbon adsorbe [22, 23]. Le calcul du nombre de sites actifs accessibles à la surface des échantillons de charbon actif s'est fait de manière théorique car il permet juste d'avoir une idée sur sa quantification. Nous n'avons pas pris en considération certains facteurs. Par exemple, le volume moyen des micropores a été déterminé en utilisant l'épaisseur moyenne des micropores. Or d'après les travaux de TSUNODA et al. [24], cette épaisseur varie d'un échantillon à l'autre et dépend de la méthode de préparation du charbon actif. Par conséquent, les micropores ne peuvent pas tous avoir le même volume et la même forme cylindrique, comme nous l'avons supposé. Nous avons également supposé que seuls les micropores adsorbent. II peut arriver que les mésopores, au lieu de laisser passer les molécules du fluide, les adsorbent également 
(molécules de diamètre correspondant à celui des mésopores). Des expériences pratiques nous permettront d'obtenir de meilleurs résultats, ceci en prenant en considération les facteurs omis.

\section{CONCLUSION}

Le présent travail nous a permis de déterminer et de comparer les propriétés adsorbantes de deux échantillons de charbons actifs COMI et COM2 à partir de l'adsorption de deux solutions. II en ressort ce qui suit :

- Pour les deux charbons actifs, les ions orthophosphates sont plus adsorbés que l'acide 5-sulfosalicylique et les charbons basiques sont plus adsorbants que les charbons neutres.

- Les calculs des sites actifs à la surface des charbons confirment les résultats expérimentaux obtenus à partir des isothermes d'adsorption, car plus le nombre de sites actifs est élevé, plus l'adsorption est forte, d'où la classification (COM2) ions > $(\mathrm{COMI})$ ions $>(\mathrm{COM} 2)$ ac $>(\mathrm{COMI})$ ac. Ce résultat est aussi confirmé par celui obtenu pour plusieurs charbons actifs préparés localement [13-15].

- Les isothermes d'adsorption étant du Type I de la classification de Langmuir, la quantité de soluté adsorbé est limitée par la porosité. Lorsque les pores sont remplis, l'adsorption n'a plus lieu que sur la surface externe des granules solides. De plus, si l'on considère que la nature chimique de la surface ne joue qu'un rôle secondaire dans l'adsorption, les propriétés adsorbantes d'un charbon actif peuvent essentiellement être attribuées à la surface spécifique et à la structure des pores. En outre, pour chaque molécule, la surface effective d'adsorption n'existe que pour des pores dans lesquels la molécule peut entrer.

- D'une façon générale, pour tout échantillon de charbon actif utilisé au travers de la relation ( I), l'influence du nombre de sites actifs accessibles à la surface des charbons actifs a été évaluée vis-à-vis de l'adsorption. II en est ressorti que plus les particules de charbon sont petites et la répartition de sites actifs accessibles élevée, plus les molécules du fluide sont fortement adsorbées, ce qui confirme les résultats de la littérature [23, 24].

En définitive, les charbons à caractère basique s'avèrent être de bons adsorbants d'ions orthophosphates et d'acide 5sulfosalicylique en solution aqueuse. Ils peuvent donc être utilisés pour les éliminer des eaux usées.

Pour des études futures, nous proposons d'étudier le mécanisme réactionnel et de préparer nos charbons actifs à partir de sousproduits végétaux, de manière à obtenir des surfaces basiques pour mieux adsorber des polluants. Toutefois, afin d'obtenir les meilleures conditions d'adsorption, une adsorption dynamique doit être effectuée pour permettre de déterminer les cinétiques d'adsorption, et certaines caractéristiques thermodynamiques et physico-chimiques doivent être prises en considération, en particulier la capacité d'adsorption maximale et la température, qui doit être aussi proche que possible de celle utilisée lors du traitement des eaux usées.

\section{Remerciements}

Nous remercions tous les membres de l'équipe «Adsorption \& Surface » du Laboratoire de chimie physique et théorique de l'Université de Yaoundé I pour leurs remarques et suggestions.

\section{Bibliographie}

I. P.W. Atkins and W.H.Freeman, Physical Chemistry, New York (1986).

2.A.W.Adamson, Physical Chemistry of Surfaces, Wiley, New York (1990).

3. G. A. Samorjai, Chemistry in Two dimensions, Cornell University, Ithaca, New York (1981).

4.W. J.Weber Jr., Physicochemical Processes for Water Quality Control,Wiley, New York (1972).

5. L. Xing, Z. Binsheng and L. Huitan, Immunotoxicity of Pesticides on Human and in Relating to Thyroid Hormones. Congrès de Yokohama, 29 juin-2 juillet, p. 202 (1997).

6. P. Dangaix, Pesticides dans l'eau potable. Généraliste, n 1782, p. 24-25 (1997)

7. M. F. Dreyfuss, M. Lotfi and G. Lachatre, Surveillance analytique de la teneur en résidus de pesticides dans les fruits et les miels. Congrès de Paris, 22 et 23 septembre, p. 19 (1994).

8T. C. Marrs, Toxicology of Pesticides. General \& Applied Toxicology. 2, I329-1 342 (1993)

8. R. R. Laurerys and P. Hoet, Organophosphorus Pesticides, Industrial Chemical Exposure Guideline for biological monotoring. Second Edition, p. 256-259 (1993).

9. L. Ford, Inc. Water Eng. 14, 20 (1977).

I0. J. Porter and T. N. Sargeant, J.Am. Soc. Text. Chem. Color, 9, 38 (1977).

I I. N. Chereminisinoff and F. Ellerbush, Carbon Adsorption Handbook., Ann Arbor Science, Michigan (1978)

12. J.Avom, J. Ketcha Mbadcam, C. Noubactep and P. Germain, Carbon, 35(3), 365 (1997).

13. J. Avom, J. Ketcha Mbadcam, M. R. L. Matip et P. Germain, African Journal of Science et Technology (A J ST), Science and Engineering Series, 2(2), I (200 I)

14. J.Avom, J. Ketcha Mbadcam, I. Ngono Abega et P. Germain, Déchets, Sciences et Technique, Revue Francophone d'Ecologie Industrielle, 28, 26 (2002).

I5.J. Basset, R. Denney, G. Jeffery and J. Mendhan.Vogel's Textbook of Quantitative Inorganic Analysis, Edition 4, p. 756 (1982)

6. J. S. Mattson and H. B. Mark, Activated Carbon, Surface Chemistry and Adsorption from Solution, Marcel Dekker Inc., New York (I97|).

17. E. P. Barett, L. Joiner and G. Halenda.J. Am. Chem. Soc. 373 (195I).

|8. M. M. Dubinin, G. M. Plavnik and E. F. Zaverina, Carbon, 2, 261 (1964).

19. J. Ketcha Mbadcam, H. Manga Ngomo, J.Avom et D.D. Dina Njoh, Déchets, Sciences \& Techniques, Revue Francophone d'Ecologie Industrielle, 36, 38 (2004).

20. G. C. Bond, Supported metal catalysts: some unsolved problems, Chem. Soc. Rev., 20 $44 \mid-475(|97|)$

21. P. Lecloirec et G. Martin, Le charbon actif, les matériaux et son utilisation dans le traitement de l'eau, J. Chim. Phys. 4, 76 (1984).

22. M. Garric, Chimie générale, Edition Dunod Université, pp. 312-314 (1976)

23. R. Tsunoda, Adsorption of water vapor on active carbon : estimation of pore width, industrial research of Kanagouwa prefeclare, Schowa-machi, Kanazawaku, Yokohama 232, Japan (1989). 